\title{
Purification of Rice Stripe Virus
}

\author{
Gang $\mathrm{Lu}^{1,2}$, Min Yao ${ }^{1,3}$, Yijun Zhou ${ }^{4}$ and Xiaorong Tao ${ }^{1, *}$
}

${ }^{1}$ Department of Plant Pathology, Nanjing Agricultural University, Nanjing, China; ${ }^{2}$ Institute of Plant Virology, Ningbo University, Ningbo, China; ${ }^{3}$ Division of Antitumor Pharmacology, State Key Laboratory of Drug Research, Shanghai Institute of MateriaMedica, Chinese Academy of Sciences, Shanghai, China; ${ }^{4}$ Institute of Plant Protection, Jiangsu Academy of Agricultural Sciences, Nanjing, China *For correspondence: taoxiaorong@njau.edu.cn

[Abstract] Although many spherical and rod-shaped plant virus purification protocols are now available, only a few protocols on filamentous plant virus purification have been published. Here, we report a protocol for large-scale purification of Rice stripe virus (RSV) from RSV-infected rice tissues. RSV virions with high infectivity were first precipitated with polyethylene glycol (PEG) followed by pelleting through primary ultracentrifugation, ultracentrifugation in a glycerol cushion and ultracentrifugation in density gradient. The purified RSV virions can not only be viewed as filamentous particles under an electron microscope, but can also be acquired by insect vector through direct injection into insect body or through membrane feeding prior to transmission to rice plants.

Keywords: Rice stripe virus, Filamentous virion, Density gradient centrifugation, Virion purification, Ultracentrifugation

[Background] Many purification protocols for spherical and rod-shaped viruses have been published (André et al., 2002; Balke et al., 2018). These protocols all rely on chemical precipitations or density gradient centrifugations. However, purification protocols for filamentous viruses are currently limited.

Rice stripe virus (RSV) is a negative-stranded RNA virus and belongs to the genus Tenuivirus, the order Bunyavirales. RSV often causes severe damages to rice productions in many East Asian countries (Whitfield et al., 2015; Liu et al., 2018). Unlike other members in the order Bunyavirales that produce spherical and enveloped virions, RSV virions are filamentous. However, RSV genome encodes a glycoprotein that is not found in purified RSV virions(Toriyama, 1986; Lu et al., 2019). RSV virions are known to carry several copies of RNA-dependent RNA polymerase (RdRp), necessary for virus replication initiation in host plants and insect vectors. Consequently, establishment of a purification method that allows to maintain active RSV RdRp is crucial for future biological assays using purified virions.

Based on a recent report from our laboratory (Lu et al., 2019), we have now developed a purification protocol suitable for producing highly infectious RSV virions. The purified virions can be used for Transmission Electron Microscopy (TEM) and virus transmission assays through insect vectors. 


\section{Materials and Reagents}

1. Pipette tips

2. Razor blades

3. Pasteur pipette

4. Sterile syringe filters (Millipore, catalog number: SLGV033RB, or equivalent)

5. Sterile $50 \mathrm{ml}$ conical tubes (Corning, catalog number: 430921 , or equivalent)

6. Ethylenediaminetetraacetic acid (EDTA) (Sigma-Aldrich, catalog number: E9884, or equivalent)

7. Polyethylene glycol (PEG) 6000 (Sigma-Aldrich, catalog number: 81255 , or equivalent)

8. Sodium chloride ( $\mathrm{NaCl}$ ) (Sigma-Aldrich, catalog number:S5886, or equivalent)

9. Glycerol (Sigma-Aldrich, catalog number: G5516, or equivalent)

10. $\mathrm{Na}_{2} \mathrm{HPO}_{4} \cdot 12 \mathrm{H}_{2} \mathrm{O}$

11. $\mathrm{NaH}_{2} \mathrm{PO} 4 \cdot 2 \mathrm{H}_{2} \mathrm{O}$

12. $\mathrm{NaOH}$

13. Coomassie Brilliant Blue G-250 (Sigma-Aldrich, catalog number: 1.15444, or equivalent)

14. Mouse anti-RSV nucleocapsid monoclonal antibody (Provided by Prof.Jianxiang $\mathrm{Wu}$ in Zhejiang University)

15. Goat anti-mouse IgG HRP conjugate (Invitrogen, catalog number: 31430 , or equivalent)

16. $0.1 \mathrm{M}$ phosphate buffer, $\mathrm{pH} 7.5$ (see Recipes)

\section{Equipment}

1. Blender (Philips, catalog number: HR2116, or equivalent)

2. $1 \mathrm{~L}$ glass beaker

3. Refrigerated centrifuge (Eppendorf, catalog number: 5810R)

4. $4{ }^{\circ} \mathrm{C}$ refrigerator (Haier, catalog number: HYC-391, or equivalent)

5. Pipettes (Eppendorf, catalog number: R42223G, or equivalent)

6. Ice machines (Phcbi, catalog number: SIM-F140AY65-PC, or equivalent)

7. Precision balances (Sartorius, model: Practum313-1CN, or equivalent)

8. Ultracentrifuge (Beckman Coulter, model: Optima XPN-90)

9. Ultra-Clear $26.3 \mathrm{ml}$ tubes for $70 \mathrm{Ti}$ rotor (Beckman Coulter, catalog number: 355618 )

10. Ultra-Clear $10.4 \mathrm{ml}$ tubes for $90 \mathrm{Ti}$ rotor (Beckman Coulter, catalog number: 355603 )

11. Ultra-Clear $10 \mathrm{ml}$ tubes for SW32.1 rotor (Beckman Coulter, catalog number: 344061)

12. Formvar and carbon coated 200 mesh Nickel grids (Electron Microscopy Sciences, catalog number: FCF200-Ni)

13. Transmission electron microscope (Hitachi, model: HT-7700)

14. ChemiDoc Touch Imaging System (Bio-Rad, catalog number: 1708370) 


\section{Procedure}

1. Laodelphaxstriatellus carrying RSV was used to transmit the virus to health rice seedling. After inoculation on rice seedling for $48 \mathrm{~h}$, the rice plants were maintained in greenhouse at $30^{\circ} \mathrm{C}$ in light ( $16 \mathrm{~h}$ ) and $26^{\circ} \mathrm{C}$ in dark $(8 \mathrm{~h})$ for 60 days. The RSV-infected rice leaves showing the typical leaf stripe symptoms were collected (Figure 1A).

2. Keep $0.1 \mathrm{M}$ phosphate buffer (PB), pH 7.5, containing 0.01 M EDTA (PB-EDTA), and a blender at $4{ }^{\circ} \mathrm{C}$.

3. Cut $50 \mathrm{~g} \mathrm{RSV-infected} \mathrm{rice} \mathrm{leaves} \mathrm{into} \mathrm{small} \mathrm{pieces} \mathrm{(about} 2 \mathrm{~cm}$ ) with razor blades and then ground them in $800 \mathrm{ml}$ chilled PB-EDTA in the chilled blender for $5 \mathrm{~min}$.

4. Filter the homogenate through two layers of gauze to remove large debris.

5. Centrifuge the filtered extract at $8,000 \times \mathrm{g}$ for $20 \mathrm{~min}$ at $4{ }^{\circ} \mathrm{C}$ to remove fine cell debris.

6. During centrifugation, weigh PEG 6000 powder (we grind PEG 6000 into fine powders before use) and $\mathrm{NaCl}$ according to the supernatant volume.

7. Transfer the supernatant into a sterilized and pre-chilled $1 \mathrm{~L}$ glass beaker.

8. Slowly add PEG 6000 and $\mathrm{NaCl}$ into the glass beaker till a final concentration of $6 \%$ PEG (w/v) and $0.1 \mathrm{M} \mathrm{NaCl}$. Stir the mixture at $4{ }^{\circ} \mathrm{C}$ for $4 \mathrm{~h}$ and then allow this mixture to stand overnight at $4{ }^{\circ} \mathrm{C}$.

9. Centrifuge this mixture at $8,000 \times g$ for $20 \mathrm{~min}$ at $4{ }^{\circ} \mathrm{C}$, using an angle rotor in a refrigerated centrifuge, to pellet RSV virions.

10. Resuspend the pellet in $8 \mathrm{ml} 0.01 \mathrm{M}$ PB-EDTA and centrifuge again at $8,000 \times \mathrm{g}$ for $10 \mathrm{~min}$ at $4{ }^{\circ} \mathrm{C}$.

11. Transfer the supernatant into a clean pre-chilled centrifuge tube. Repeat Step 9 twice.

12. Combine the three supernatant in a pre-chilled ultra-centrifuge tube for the 70Ti rotor and centrifuge at $150,000 \times g$ for $2 \mathrm{~h}$ at $4{ }^{\circ} \mathrm{C}$. Discard the supernatant and resuspend the pellet in $6 \mathrm{ml} 0.01 \mathrm{M} \mathrm{PB}$.

13. Add $4 \mathrm{ml} 20 \%$ glycerol solution into a clean ultra-clear centrifuge tube for the $90 \mathrm{Ti}$ rotor to form a glycerol cushion.

14. Carefully lay the resuspended pellet solution on top of the glycerol cushion and centrifuge at $150,000 \times g$ for $2 \mathrm{~h}$ at $4{ }^{\circ} \mathrm{C}$.

15. After centrifugation (Figure 1B), discard the supernatant and resuspend the pellet with $4 \mathrm{ml} P B$ through repeated pipetting.

16. Prepare $10 \%, 20 \%, 30 \%$ and $40 \%$ sucrose solutions. $2 \mathrm{ml} 40 \%$ sucrose solution was firstly added into the bottom of ultra-centrifuge tube and then slowly add $2 \mathrm{ml}$ each of $30 \%, 20 \%$ and $10 \%$ sucrose solutions, sequentially, on the top of $10 \%$ sucrose solution with pipettes.

17. Carefully lay the resuspended pellet solution on a $10-40 \%$ sucrose density gradient inside a clean ultra-centrifuge tube for the SW32.1 rotor and centrifuge at $70,000 \times g$ for $2 \mathrm{~h}$ at $4{ }^{\circ} \mathrm{C}$.

18. Place the centrifuge tube under a light source and two separate bands become visible (Figure 1C, $M$ and B). Collect the two bands separately and analyze them by SDS-PAGE (Figure 2A), 
Western blot (Figure 2B) and TEM (Figure 3). Add glycerol into the purified virion sample (M band) to reach a final concentration of $30 \%$. Store the sample at $-70{ }^{\circ} \mathrm{C}$ till further use.
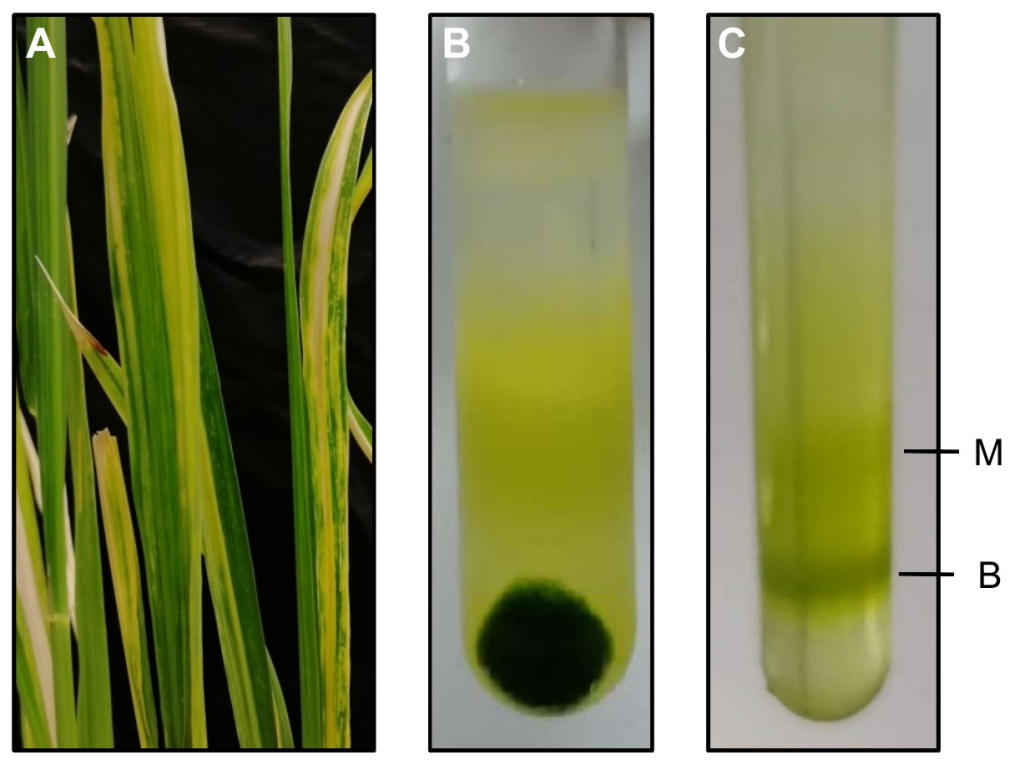

Figure 1. Purification of RSV through ultracentrifugation from RSV-infected rice leaves. A. RSV-infected rice samples showing typical stripe symptoms. B. Pelleting RSV virions through $20 \%$ glycerol cushion ultracentrifugation. A dark-green pellet with RSV virions is at the bottom of the centrifuge tube. C. Purification of RSV virions through $10-40 \%$ sucrose density gradient ultracentrifugation. The middle and bottom bands with RSV virions are indicated. M, middle band; $\mathrm{B}$, bottom band.

\section{Data analysis}

1. SDS-PAGE

$M$ and $B$ bands are collected separately and analyzed by SDS-PAGE (Figure 2A). Small amounts of samples from the $M$ and $B$ bands are respectively mixed with a loading buffer and boiled at $95{ }^{\circ} \mathrm{C}$ for $10 \mathrm{~min}$. These samples are separated in lanes of a 10\% SDS-PAGE gel through 50 min electrophoresis at $120 \mathrm{~V}$. The gel is stained with $0.5 \%$ Coomassie Brilliant Blue G-250 before imaging.

2. Western blot

$M$ and $B$ components are further analyzed by Western blot (Figure 2B). After separated by $10 \%$ SDS-PAGE through electrophoresis, the proteins are transferred onto nitrocellulose membrane and the blot was probed with a 1:5,000 (v/v) diluted mouse anti-RSV nucleocapsid monoclonal antibody followed by a goat anti-mouse IgG conjugated with HRP. The blot is visualized using the ChemiDoc Touch Imaging System. 
A

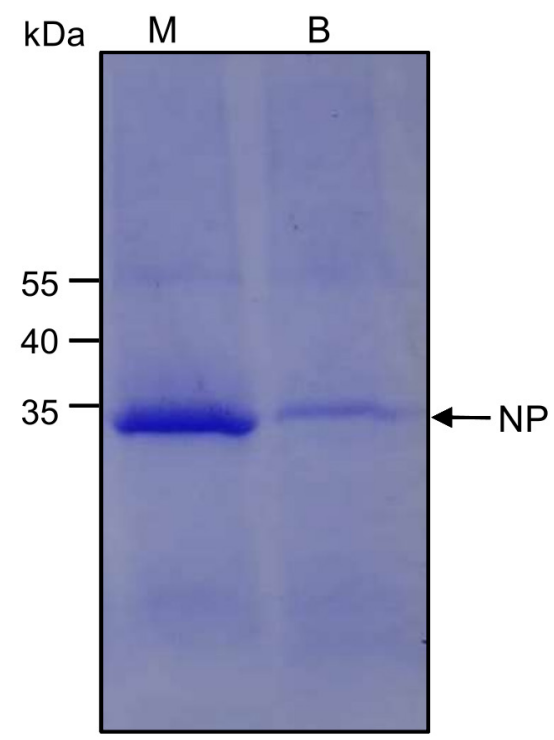

B

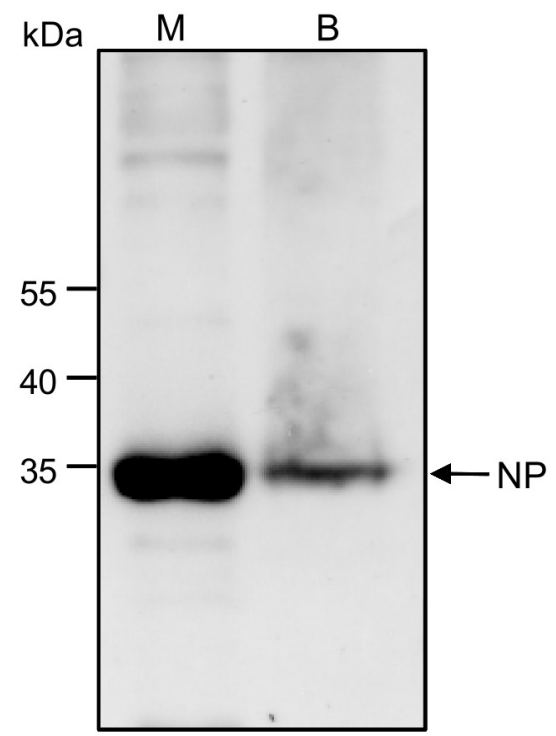

Figure 2. Analysis of $M$ and $B$ band products by SDS-PAGE and Western blot. A. After centrifugation in a $10-40 \%$ sucrose density gradient, the $M$ and $B$ bands are collected separately and analyzed in a 10\% SDS-PAGE gel through electrophoresis. The gel is stained with Coomassie blue G-250. The band with predicted size for the RSV nucleocapsid protein (NP) is indicated with an arrow.B. Western blot analysis of $M$ and $B$ bands using monoclonal antibody against RSV NP. A band corresponding to the RSV nucleocapsid protein (NP) is indicated with an arrow.

\section{Transmission Electron Microscopy}

For TEM, purified RSV sample is diluted 1:50 (v/v) 0.01 M PB and then placed ( $5 \mu$ per sample) one formvar and carbon coated 200 mesh nickel grid. One minute after, the sample is removed from the grid by touching the edge of the grid with a filter paper. The grid is then stained with a $1 \%$ uranyl acetate solution for $2 \mathrm{~min}$. After removal of the uranyl acetate solution, the grid is examined under an electron microscope. RSV virionsare only detected in $\mathrm{M}$ band sample (Figure 3). 


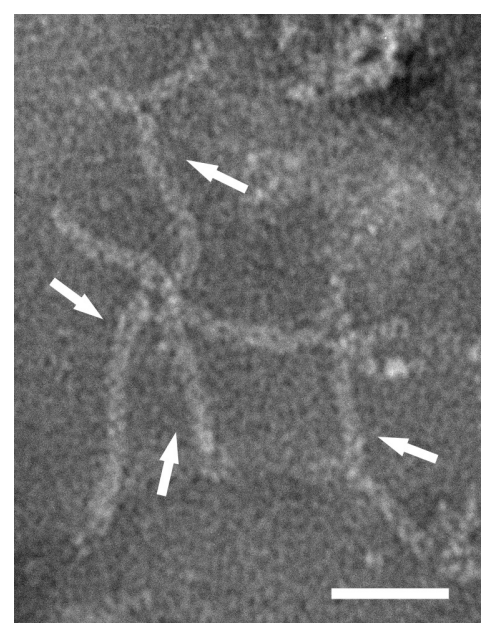

Figure 3. A TEM image showing RSV virions. RSV virions are purified from a RSV-infected rice plant using the above protocol, stained with uranyl acetate solution, and then examined and imaged under an electron microscope. Arrows indicate filamentous RSV virions. Scale bar $=50 \mathrm{~nm}$.

\section{$\underline{\text { Notes }}$}

1. To avoid degradation of virions, each purification step should be done at about $4{ }^{\circ} \mathrm{C}$. The purified virion sample should be immediately stored at $-70^{\circ} \mathrm{C}$.

2. Glycerol can affect RSV visualization under an electron microscope. We recommend to use freshly purified RSV for TEM without addition of glycerol.

\section{Recipes}

1. $0.1 \mathrm{M}$ phosphate buffer, $\mathrm{pH} 7.5$

a. Dissloved $21.2 \mathrm{~g} \mathrm{Na}_{2} \mathrm{HPO}_{4} \cdot 12 \mathrm{H}_{2} \mathrm{O}$ and $2.21 \mathrm{~g} \mathrm{NaH}_{2} \mathrm{PO}_{4} \cdot 2 \mathrm{H}_{2} \mathrm{O}$ in $1 \mathrm{~L}$ distilled and sterilized water

b. Adjust $\mathrm{pH}$ value to 7.5 using $\mathrm{NaOH}$

c. Store the solution at $4{ }^{\circ} \mathrm{C}$ refrigerator

\section{Acknowledgments}

We thank Prof. Tong Zhou (Institute of Plant Protection, Jiangsu Academy of Agricultural Sciences) for providing RSV-infected rice plants. This work is supported by the National Natural Science Foundation of China (31925032, 31630062 and 31870143), the Fundamental Research Funds for the Central Universities (JCQY201904) and sponsored by K.C.Wong Magna Fund in Ningbo University. 


\section{Competing interests}

The authors declare no conflicts of interest or competing interests.

\section{$\underline{\text { References }}$}

1. André, P., Komurian-Pradel, F., Deforges, S., Perret, M., Berland, J. L., Sodoyer, M., Pol, S., Brechot, C., Paranhos-Baccala, G. and Lotteau, V. (2002). Characterization of low- and very-low-density hepatitis C virus RNA-containing particles. J Virol 76(14): 6919-6928.

2. Balke, I., Resevica, G. and Zeltins, A. (2018). Isolation and characterization of two distinct types of unmodified spherical plant sobemovirus-like particles for diagnostic and technical uses. Methods MolBiol 1776: 19-34.

3. Liu, W. W., Hajano, J. U. D. and Wang, X. F. (2018). New insights on the transmission mechanism of tenuiviruses by their vector insects. Curr Opin Virol 33: 13-17.

4. Lu, G., Li, S., Zhou, C., Qian, X., Xiang, Q., Yang, T., Wu, J., Zhou, X., Zhou, Y., Ding, X. S. and Tao, X. (2019). Tenuivirus utilizes its glycoprotein as a helper component to overcome insect midgut barriers for its circulative and propagative transmission. PLoS Pathog 15(3): e1007655.

5. Toriyama, S. (1986). An RNA-dependent RNA-polymerase associated with the filamentous nucleoproteins of rice stripe virus. J Gen Virol 67: 1247-1255.

6. Whitfield, A. E., Falk, B. W. and Rotenberg, D. (2015). Insect vector-mediated transmission of plant viruses. Virology 479-480: 278-289. 